\title{
An isogeometric BEM for exterior potential-flow problems in the plane.
}

\author{
Costas Politis \\ TEI of Athens* \\ Dept Naval Architecture \\ cpolitis@ teiath.gr \\ Alexandros I. Ginnis \\ NTUA-SDL ${ }^{\dagger}$ \\ CAGD/CAD/VR Group \\ ginnis@ naval.ntua.gr
}

\author{
Panagiotis D.Kaklis \\ NTUA - SDL \\ CAGD/CAD/VR Group \\ kaklis@deslab.ntua.gr
}

\author{
Kostas Belibassakis \\ TEI of Athens \\ Dept Naval Architecture \\ kbel@teiath.gr
}

\author{
Christian Feurer \\ NTUA - SDL \\ CAGD/CAD/VR Group \\ christian_feurer@naval.ntua.gr
}

\begin{abstract}
In this paper, the isogeometric concept introduced by Hughes, in the context of Finite Element Method, is applied to Boundary Element Method (BEM), for solving an exterior planar Neumann problem. The developed isogeometric-BEM concept is based on NURBS, for representing the exact body geometry and employs the same basis for representing the potential and/or the density of the single layer. In order to examine the accuracy of the scheme, numerical results for the case of a circle and a free-form body are presented and compared against analytical solutions. This enables performing a numerical error analysis, verifying the superior convergence rate of the isogeometric BEM versus low-order BEM. When starting from the initial NURBS representation of the geometry and then using knot insertion for refinement of the NURBS basis, the achieved rate of convergence is $O\left(D_{o F}^{-4}\right)$. This rate may be further improved by using a degree-elevated initial NURBS representation of the geometry ( $k h$-refinement).
\end{abstract}

Keywords: isogeometric analysis (IGA), Boundary Element Methods (BEM), collocation method, exterior potential flows, NURBS

\section{Introduction}

Integral equation method has been established as one of the standard tools for solving boundary value problems, e.g., inviscid, incompressible flow around complex 2-D and 3-D bodies and geometrical systems; see, e.g., [Jawson and Symm 1977], [Kress 1989]. Some of the most important advantages of this approach are: i) reduction of the dimensionality of the problem by one, facilitating calculations around complex geometrical configurations (especially in 3-D problems) and ii) consistent treatment of conditions at infinity. As regards the numerical solution of the integral equations, Boundary Element Methods (BEM) serve today as the main tool, [Hess 1975], [Brebbia et al. 1984], [Paris and Canas 1997], [Brebbia 2002], although various other techniques are also available, such as spectral methods using basis functions of global support on the boundary surface.

In the low-order BEM, the body surface is usually discretized by a finite number of linear elements or patches, each carrying a constant distribution of the unknown function (potential, sources and/or doublets). The body-boundary condition is then applied to the centroid of the linear elements. Analytical or semi-analytical expressions for the dependent quantities permit fast calculation of the co-

\footnotetext{
*TEI: Technological Educational Institute

${ }^{\dagger}$ NTUA: National Technical University of Athens - SDL: Ship Design Laboratory
}

efficient matrix, discretizing the integral equation. In the case of regular or weakly singular integral equations of the second-kind, which is also our case (eq. (8)), the matrix is diagonally dominant and the linear system may be rapidly solved by iterative methods. In 2D problems, the overall accuracy of low-order BEM is $O(h)$, where $h$ denotes the element size, see, e.g., [Schmidt and Strese 2001]) and, since $h$ is inversely proportional to the degreesof-freedom, $D o F$, (number of elements), the rate of convergence becomes $O\left(D o F^{-1}\right)$.

Despite of its low rate of convergence, this method has been widely employed in practical applications due to its easy implementation, in conjunction with the accuracy obtained by using a quite large number of elements. However, there exist situations where BEM may still pose considerable challenges, both from the modeling and the computational point of view. BEM solutions in these cases require the use of accurate and efficient numerical algorithms, which need to be applied on extremely fine meshes. Such cases arise in the context of non-linear and/or time-dependent problems, as, e.g., problems with free boundaries and/or inverse problems; see, e.g., [Krishnaswamy et al. 2001]. In this context, higher-order BEM, characterized by an increased order of approximation both with respect to geometry and the dependent quantities, may become an attractive approach to further increase the convergence rate and reduce computational time accordingly. In these cases, depending on the smoothness of the body boundary, as well as the singularity of the solution, BEM may attain rates of convergence up to $O\left(D o F^{-4}\right)$, [Vaz et al. 2003].

In the present work, the isogeometric concept proposed by Hughes and collaborators, [Hughes et al. 2005], [Hughes (to appear)], see also, [Cottrell et al. 2007], in the context of Finite Element Method is applied to BEM. The primary goal of the isogeometric concept is to be geometrically exact, no matter how coarse the discretisation of the geometry is. A second goal is to simplify mesh refinement, by eliminating the need for communication with the CAD tool once the initial mesh is constructed. This is usually achieved by adopting NURBS basis to construct an exact geometric model. Invoking then the isoparametric philosophy, the solution space for the dependent variables (e.g., source density, potential) is represented in terms of the very same NURBS basis. For the purposes of analysis, this basis can be easily refined using knot insertion and/or degree elevation, without changing the geometry or its parametrization.

In order to examine the accuracy of the proposed isogeometricBEM concept, presented in $\S 3$, we employ it to solve a classical exterior Neumann problem $(\S 2)$ and test it numerically for the case of a circle and a free-form body $(\S 4)$. The obtained numerical results are compared against analytical solutions, which enables performing a numerical-error analysis illustrating the superior convergence rate of the proposed isogeometric method versus low-order 
BEM. When using knot insertion for refinement, the achieved rates are, in general, $O\left(D o F^{-4}\right)$, which may be further increased up to $O\left(D o F^{-9}\right)$ by $k h$-refinement; see [Cottrell et al. 2007].

\section{The model problem}

Let be given the following exterior boundary-value problem: find a function $\tilde{\Phi}(\mathbf{x})$ belonging to $C^{2}(\mathcal{D}) \cap C^{1}(\overline{\mathcal{D}})$, which satisfies the following conditions:

$$
\begin{aligned}
& \triangle \tilde{\Phi}(\mathbf{x})=0, \quad \mathbf{x}=\left(x_{1}, x_{2}\right) \in \mathcal{D}, \\
& \frac{\partial \tilde{\Phi}(\mathbf{x})}{\partial \mathbf{n}}=\tilde{g}(\mathbf{x}), \quad \mathbf{x} \in \partial \mathcal{D}, \\
& \tilde{\Phi}(\mathbf{x})=o(1) \text { as }\|\mathbf{x}\| \rightarrow \infty \text { uniformly } \\
& \text { for all directions } \mathbf{x} /\|\mathbf{x}\|,
\end{aligned}
$$

where $\triangle$ denotes the Laplace operator, $\mathcal{D}$ is an unbounded planar domain lying in the exterior of a Jordan curve $\partial \mathcal{D}, \mathbf{n}=\left(n_{1}, n_{2}\right)$ denotes the unit normal vector on $\partial \mathcal{D}$, pointing inwards with respect to $\mathcal{D}$ and, finally, $\tilde{g}(\mathbf{x})$ is a given function belonging to $C^{1}(\partial \mathcal{D})$.

The problem (1)-(3) is a classical exterior planar Neumann problem with applications in steady-state potential problems in fluid dynamics, electromagnetism, heat conduction etc. In order to ensure unique solvability it is necessary and sufficient that the following condition should be fulfilled

$$
\int_{\partial \mathcal{D}} \tilde{g}(\mathbf{x}) d s=0
$$

where $d s$ denotes the differential arc length on the boundary $\partial \mathcal{D}$. Assuming now that $\partial \mathcal{D}$ is adequately smooth, that is of class $C^{2}$, we can obtain an integral representation of the potential $\tilde{\Phi}(\mathbf{x})$, $\mathbf{x} \in \overline{\mathcal{D}}$, by means of a single-layer potential on $\partial \mathcal{D}$ with continuously differentiable density $\tilde{\mu}(\mathbf{x})$, expressed as follows:

$$
\tilde{\Phi}(\mathbf{x})=\int_{\partial \mathcal{D}} \tilde{\mu}(\xi) \tilde{G}(\xi, \mathbf{x}) d s_{\xi}, \quad \mathbf{x} \in \overline{\mathcal{D}}
$$

where

$$
\tilde{G}(\xi, \mathbf{x})=\frac{1}{2 \pi} \ln \|\mathbf{x}-\xi\|, \quad \xi \in \partial \mathcal{D}, \mathbf{x} \in \overline{\mathcal{D}}
$$

is the fundamental solution of the Laplace equation in the plane. Using now representation (5) in conjunction with the jump properties of the normal derivative of the single-layer potential as $\mathbf{x} \in \mathcal{D}$ tends to $\mathbf{x}_{0} \in \partial \mathcal{D}$, namely

$$
\lim _{\mathbf{x} \rightarrow \mathbf{x}_{0}} \frac{\partial \tilde{\Phi}(\mathbf{x})}{\partial \mathbf{n}}-\frac{\partial \tilde{\Phi}\left(\mathbf{x}_{0}\right)}{\partial \mathbf{n}}=\frac{1}{2} \tilde{\mu}\left(\mathbf{x}_{0}\right),
$$

the initial boundary-value problem (1)-(3) is reduced to the following Fredholm's integral equation of the second kind:

$$
\frac{1}{2} \tilde{\mu}(\mathbf{x})+\int_{\partial \mathcal{D}} \tilde{\mu}(\xi) \tilde{K}(\xi, \mathbf{x}) d s_{\xi}=\tilde{g}(\mathbf{x}), \quad \mathbf{x} \in \partial \mathcal{D}
$$

where the kernel function

$$
\tilde{K}(\xi, \mathbf{x})=\frac{\partial}{\partial \mathbf{n}_{\mathbf{x}}} \tilde{G}(\xi, \mathbf{x})=\frac{1}{2 \pi} \frac{\mathbf{x}-\xi}{\|\mathbf{x}-\xi\|^{2}} \cdot \mathbf{n}_{\mathbf{x}}
$$

is continuous with respect to both $\mathbf{x}$ and $\xi$ on $\partial \mathcal{D}$.

\section{The isogeometric-BEM concept}

As pointed out in the introduction, the purpose of this section is to present a method that combines BEM with Isogeometric Analysis (IGA) for solving the boundary integral equation (8). IGA philosophy is equivalent to approximating the field quantities (dependent variables) of the boundary-value problem in question by the very same basis that is being used for representing accurately the geometry of the involved body-boundary. In the case of the boundary integral equation (8), which we are interested in, the dependent variable is the density $\tilde{\mu}(\mathbf{x}), \mathbf{x} \in \partial \mathcal{D}$, of the logarithmic singularities distributed over the contour $\partial \mathcal{D}$. For the latter, we shall presume that $\partial \mathcal{D}$ can be accurately represented as a regular parametric NURBS curve $\mathbf{r}(t), t \in I$. In this connection it should be stressed that, although NURBS is not a requisite IGA ingredient, we adopt it since it constitutes a standard representation in CAD industry. So, let

$$
\mathbf{r}(t):=\sum_{i=0}^{n} \mathbf{d}_{i} M_{i k}(t), \quad t \in I=\left[t_{k-1}, t_{n+1}\right]
$$

where $\left\{M_{i k}(t)\right\}_{i=0}^{n}$ is a rational $B$-spline basis of order $k$, defined over a knot sequence $\mathcal{J}=\left\{t_{0}, t_{1}, \ldots, t_{n+k}\right\}$ and possessing non-negative weights $w_{i}, i=0,1, \ldots, n$; [Piegl and Tiller 1997]. Equation (8) can then be written in the following form:

$$
\mathcal{L}(\mu(t)):=\frac{1}{2} \mu(t)+\int_{I} \mu(\tau) K(\tau ; t)\|\dot{\mathbf{r}}(\tau)\| d \tau=g(t), \quad t \in I,
$$

where

$\mu(t):=\tilde{\mu}(\mathbf{r}(t)), \quad K(\tau ; t):=\tilde{K}(\mathbf{r}(\tau) ; \mathbf{r}(t)), \quad g(t):=\tilde{g}(\mathbf{r}(t))$.

Aiming to employ IGA for handling equation (11), we project, in a suitably defined manner, the unknown function $\mu(t)$ on the spline space $\mathcal{S}^{k}\left(\mathcal{J}^{(\ell)}\right), \mathcal{S}^{k}\left(\mathcal{J}^{(0)}\right):=\mathcal{S}^{k}(\mathcal{J})$. This projection is formally expressed in the form:

$$
\mu_{s}(t):=\mathcal{P}_{s}(\mu(t))=\sum_{i=0}^{n+\ell} \mu_{i} M_{i k}^{(\ell)}(t), \quad t \in I, M_{i k}^{(0)}:=M_{i k},
$$

where $\ell \in \mathbb{N}_{0}$ denotes the number of knots in $I$ inserted in $\mathcal{J}$. Recalling the fundamental property of knot insertion, we can say that $\left\{\mathcal{S}^{k}\left(\mathcal{J}^{(\ell)}\right), \ell \in \mathbb{N}_{0}\right\}$ constitutes a sequence of nested finite dimensional-spaces, i.e., $\mathcal{S}^{k}\left(\mathcal{J}^{(\ell)}\right) \subset \mathcal{S}^{k}\left(\mathcal{J}^{(\ell+1)}\right)$. Equation (11) can then be written as

$$
\mathcal{L}\left(\mu_{s}(t)\right)+\mathcal{L}\left(e_{\mu}(t)\right)=g(t),
$$

with $e_{\mu}(t)=\mu(t)-\mu_{s}(t)$ being the error between the accurate solution $\mu(t)$ of (11) and its spline approximation $\mu_{s}(t)$. Let us now assume that the forcing term $g(t)$ in (14) is also approximated by an element in $\mathcal{S}^{k}\left(\mathcal{J}^{(\ell)}\right)$, say:

$$
g_{s}(t):=\mathcal{P}_{s}(g(t))=\sum_{i=0}^{n+\ell} g_{i} M_{i k}^{(\ell)}(t), \quad t \in I .
$$

Adding and subtracting $g_{s}(t)$ in the right-hand side of (14), we get

$$
\left[\mathcal{L}\left(\mu_{s}(t)\right)-g_{s}(t)\right]+\left[\mathcal{L}\left(e_{\mu}(t)\right)-e_{g}(t)\right]=0,
$$

with $e_{g}(t)=g(t)-g_{s}(t)$. Using the fact that $\mu_{s}(t), g_{s}(t) \in$ $\mathcal{S}^{k}\left(\mathcal{J}^{(\ell)}\right)$ (see eqs. (13) and (15)), the first bracket term in (16) takes the following form:

$\mathcal{L}\left(\mu_{s}(t)\right)-g_{s}(t)=\sum_{i=0}^{n+\ell}\left(\frac{1}{2} \mu_{i} M_{i k}^{(\ell)}(t)+\mu_{i} q_{i}(t)-g_{i} M_{i k}^{(\ell)}(t)\right)$, 
where

$$
q_{i}(t)=\int_{I} M_{i k}^{(\ell)}(\tau) K(\tau, t)\|\dot{\mathbf{r}}(\tau)\| d \tau
$$

We now proceed to approximate the kernel terms $q_{i}(t)$ through the same spline space $\mathcal{S}^{k}\left(\mathcal{J}^{(\ell)}\right)$, namely

$$
q_{i s}(t):=\mathcal{P}_{s}\left(q_{i}(t)\right)=\sum_{j=0}^{n+\ell} q_{i j} M_{j k}^{(\ell)}(t) .
$$

Adding and subtracting $q_{i s}(t)$ in the $q_{i}$-term in the right-hand side of (17), the latter is split into two terms as follows:

$$
\begin{aligned}
& \mathcal{L}\left(\mu_{s}(t)-g_{s}(t)\right)=\sum_{i=0}^{n+\ell} \mu_{i}\left(q_{i}(t)-q_{i s}(t)\right)+ \\
& +\sum_{i=0}^{n+\ell}\left(\frac{1}{2} \mu_{i} M_{i k}^{(\ell)}(t)+\mu_{i} q_{i s}(t)-g_{i} M_{i k}^{(\ell)}(t)\right) .
\end{aligned}
$$

Substituting (19) into the second term of the right-hand side of (20), we arrive after some straightforward calculations at the expression below:

$$
\begin{aligned}
& \sum_{i=0}^{n+\ell}\left(\frac{1}{2} \mu_{i} M_{i k}^{(\ell)}(t)+\mu_{i} q_{i s}(t)-g_{i} M_{i k}^{(\ell)}(t)\right)= \\
& \sum_{j=0}^{n+\ell}\left[\frac{1}{2} \mu_{j}+\sum_{i=0}^{n+\ell} \mu_{i} q_{i j}-g_{j}\right] M_{j k}^{(\ell)}(t) .
\end{aligned}
$$

Combining (16) with (20) and (21), the first relation becomes

$$
\begin{gathered}
\sum_{j=0}^{n+\ell}\left[\frac{1}{2} \mu_{j}+\sum_{i=0}^{n+\ell} \mu_{i} q_{i j}-g_{j}\right] M_{j k}^{(\ell)}(t)+ \\
\sum_{j=0}^{n+\ell} \mu_{j}\left(q_{j}(t)-q_{j s}(t)\right)+\mathcal{L}\left(e_{\mu}(t)\right)-e_{g}(t)=0 .
\end{gathered}
$$

Summarizing the work done so far, we can say that (22) provides an equivalent formulation of the integral equation (11) that employs IGA approximations of the density $\mu(t)$ as well as the kernel moments $q_{i}(t)$ and the forcing term $g(t)$ through the NURBS space $\mathcal{S}^{k}\left(\mathcal{J}^{(\ell)}\right)$. This formulation will be used in the sequel to derive the two standard approximation approaches in BEM practice, namely the Galerkin and the collocation method, [Brebbia et al. 1984]. For this purpose, we rewrite (22) as

$$
\begin{array}{r}
\sum_{j=0}^{n+\ell}\left[\frac{1}{2} \mu_{j}+\sum_{i=0}^{n+\ell} \mu_{i} q_{i j}-g_{j}\right] M_{j k}^{(\ell)}(t)+ \\
\sum_{j=0}^{n+\ell} \mu_{i}\left(q_{j}(t)-q_{j s}(t)\right)-e_{g}(t)=\mathcal{L}\left(-e_{\mu}(t)\right)
\end{array}
$$

We proceed by firstly devising the Galerkin technique, that is equivalent to enforcing the projection of the residual error $\mathcal{L}\left(-e_{\mu}(t)\right.$ onto $\mathcal{S}^{k}\left(\mathcal{J}^{(\ell)}\right)$ to vanish, i.e.,

$$
\mathcal{P}_{s}\left(\mathcal{L}\left(e_{\mu}(t)\right)\right)=0
$$

Using (23) and taking into account that the projections of $q_{j}(t)-$ $q_{j s}(t)$ and $e_{g}(t)$ vanish, (24) gives

$$
\sum_{j=0}^{n+\ell}\left[\frac{1}{2} \mu_{j}+\sum_{i=0}^{n+\ell} \mu_{i} q_{i j}-g_{j}\right] M_{j k}^{(\ell)}(t)=0
$$

which, due to the basis property of $\left\{M_{j k}^{(\ell)}(t)\right\}_{i=0}^{n+\ell}$, is equivalent to the linear system for $\mu_{j}$ :

$$
\frac{1}{2} \mu_{j}+\sum_{i=0}^{n+\ell} \mu_{i} q_{i j}=g_{j}, \quad j=0, \ldots, n+\ell .
$$

The collocation technique is obtained by enforcing $\mathcal{L}\left(-e_{\mu}(t)\right)$ to vanish over a set of parametric values $t=\hat{t}_{i}, i=0, \ldots, n+\ell$. Applying these constraints on (14) we get

$$
\mathcal{L}\left(-e_{\mu}\left(\hat{t}_{i}\right)\right)=0=\mathcal{L}\left(\mu_{s}\left(\hat{t}_{i}\right)\right)-g\left(\hat{t}_{i}\right), \quad i=0, \ldots, n+\ell,
$$

which, with the aid of (11) and (18), yields the following linear system for the unknown coefficients $\mu_{j}$ :

$$
\sum_{j=0}^{n+\ell}\left(\frac{1}{2} M_{j k}^{(\ell)}\left(\hat{t}_{i}\right)+q_{j}\left(\hat{t}_{i}\right)\right) \mu_{j}=g\left(\hat{t}_{i}\right), \quad i=0, \ldots, n+\ell .
$$

We shall now show that in a specific context the collocation, (28), and the Galerkin, (26), methods coincide. If, instead of (14), we appeal to (23) and impose the collocation constraints at $t=\hat{t}_{i}$, we can write:

$$
\begin{aligned}
& \sum_{j=0}^{n+\ell}\left[\frac{1}{2} \mu_{j}+\sum_{i=0}^{n+\ell} \mu_{i} q_{i j}-g_{j}\right] M_{j k}^{(\ell)}\left(\hat{t}_{i}\right)+ \\
& \sum_{j=0}^{n+\ell} \mu_{i}\left(q_{j}\left(\hat{t}_{i}\right)-q_{j s}\left(\hat{t}_{i}\right)\right)-e_{g}\left(\hat{t}_{i}\right) \\
& =\mathcal{L}\left(-e_{\mu}\left(\hat{t}_{i}\right)\right)=0, \quad i=0, \ldots, n+\ell
\end{aligned}
$$

If we further demand for the kernel moments (18) and the forcing $g(t)$ to be projected on $\mathcal{S}^{k}\left(\mathcal{J}^{(\ell)}\right)$ through interpolation at the collocation nodes $\hat{t}_{i}$, namely:

$$
q_{j s}\left(\hat{t}_{i}\right)=q_{j}\left(\hat{t}_{i}\right), \quad e_{g}\left(\hat{t}_{i}\right)=0, \quad i=0, \ldots n+\ell,
$$

then (29) gives:

$$
\sum_{j=0}^{n+\ell} \hat{\mu}_{j} M_{j k}^{(\ell)}\left(\hat{t}_{i}\right)=0, \quad \hat{\mu}_{j}=\frac{1}{2} \mu_{j}+\sum_{i=0}^{n+\ell} \mu_{i} q_{i j}-g_{j} .
$$

In the case where the collocation nodes $\hat{t}_{i}$ are chosen to be the Greville abscissae, see [Farin 2001] $\S 8.6$, associated with the knot vector $\mathcal{J}^{(\ell)}$, then standard spline-interpolation theory secures that $M_{j k}^{(\ell)}\left(\hat{t}_{i}\right)$ is an invertible matrix and thus $\hat{\mu}_{j}=0, j=0, \ldots n+\ell$, which is the Galerkin linear system (26). Summarizing we can say that, under the interpolation conditions (30) and the invertibility assumption on the matrix $M_{j k}^{(\ell)}\left(\hat{t}_{i}\right)$, the Galerkin and collocation techniques are equivalent.

Once we possess an approximate solution $\mu_{s}(t)$ of (11), we are faced with the task of approximating the velocity potential $\tilde{\Phi}(\mathbf{x})$ for any $\mathbf{x} \in \overline{\mathcal{D}}$, via its integral representation formula (5). The latter takes the following form with the aid of NURBS representation (10) of $\partial \mathcal{D}$ :

$$
\tilde{\Phi}(\mathbf{x})=\int_{i} \mu(\tau) \tilde{G}(\mathbf{x}, \mathbf{r}(\tau))\|\dot{\mathbf{r}}(\tau)\| d \tau, \quad \mathbf{x} \in \overline{\mathcal{D}}
$$

The integrand in the above expression is regular except if $\mathbf{x}$ lies on the body boundary $\partial \mathcal{D}$, in which case it exhibits a logarithmic singularity. In this case, the integral representation of $\Phi(t):=\tilde{\Phi}(\mathbf{r}(t))$ 
can take the following form

$$
\begin{aligned}
& \Phi(t)=\int_{I}[m(\tau)-m(t)] G(\tau, t) d \tau+m(t) \in \hat{t}_{i} G(\tau, t) d \tau, \\
& G(\tau, t):=\tilde{G}(\mathbf{r}(\tau), \mathbf{r}(t)), m(t)=\mu(t)\|\dot{\mathbf{r}}(\tau)\| .
\end{aligned}
$$

The first integral in (33) is obviously regular, while the second one, which retains the logarithmic singularity, depends exclusively on $\partial \mathcal{D}$. As a consequence, it has to evaluated only once, on the preprocessing level, appealing to standard numerical quadrature techniques for weakly singular integrals; see, e.g., [Sladek et al. 2001].

\section{Numerical Results}

In order to illustrate the efficiency and the robustness of the isogeometric approach, developed in $\S 3$, we shall present numerical results for two geometric configurations using the collocation technique as expressed by the linear system (28). The collocation nodes $\hat{t}_{i}$ are chosen to be the Greville abscissae associated with the knot vector $\mathcal{J}^{(\ell)}$, a choice inferred from the well known fact that the matrix $M_{j k}^{(\ell)}\left(\hat{t}_{i}\right)$ is totally positive. The regular character of the kernel function $K(\tau ; t)$ justifies the usage of Simpson numerical integration to calculate the coefficients $q_{i}\left(t_{j}\right)$ (see eq. (18)).

The first example concerns the flow around a unit-radius circle with center at $(0,0)$. The flow is induced from a localized Rankine-source singularity located at the point $(1,1)$. This example may be considered simplistic, however, the boundary data could become quite steep by moving the point source close to the boundary, which may affect the convergence of the numerical scheme.

Using the circle theorem [Milne-Thomson 1956], the complex potential of the flow in question becomes

$$
w(z)=f(z)+\bar{f}\left(\frac{a^{2}}{z}\right),
$$

where $f(z)=-m \log (z-\zeta)$ is the complex potential of a point source of strength $m$ located at the point $\zeta,|\zeta|>a$, with $a$ denoting the radius of the circle and $\bar{f}$ being the complex conjugate of $f$. Also note that, when $\partial \mathcal{D}$ is a circle, integral equation (11) admits an analytic solution, namely

$$
\mu(t)=2 g(t), t \in I .
$$

For the needs of our isogeometric scheme, the circle is represented as a quadratic NURBS curve with knot sequence: $\mathcal{J}=\{0,0,0$, $1 / 4,1 / 2,1 / 2,3 / 4,1,1,1\}$, control points: $[(1,0),(1,1),(-1,1)$, $(-1,0),(-1,-1),(1,-2),(1,0)]$ and weights: $[1,1 / 2,1 / 2,1$, $1 / 2,1 / 2,1]$. Knot insertion ( $h$-refinement) is performed by adding equidistant knots in the initial four non-degenerate knot intervals.

In Figure 1 the analytic disturbance potential $\Phi(t)=\operatorname{Re}\left(\bar{f}\left(a^{2} / z\right)\right)$, provided by (34) (dashed line), is compared against successive IGA approximations by using $h$-refinement up to 4 additional knots in each non-empty subinterval of $I$, as partitioned by the knot vector $\mathcal{J}$. By adding one more knot we get an approximation nondistinguishable, in the scale of Fig. 1, from the analytic solution.

In Figure 2 we compare isogeometric versus low-order BEM. This is made by plotting, for both methods, the $L^{\infty}$-norm of the error (distance between the analytic solution and the IGA approximation) of the potential on the circular boundary as a function of the degrees of freedom $(D o F)$. In all numerical results presented in this section, $L^{\infty}$-norm is approximated by sampling the error at 1000 equidistant points on $I$. In the case of low-order BEM, DoF is the number of the linear elements, whereas in the isogeometric

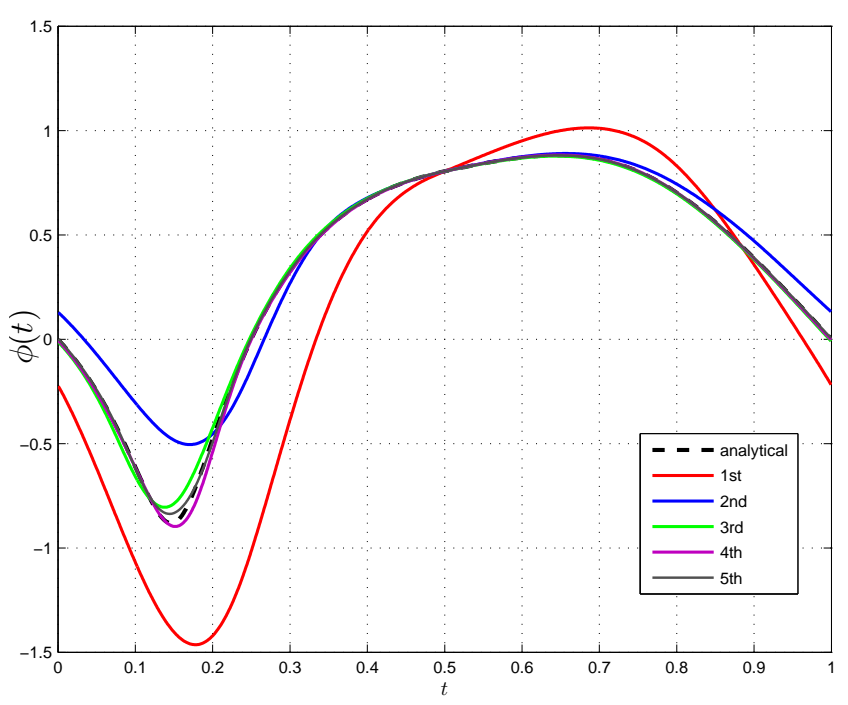

Figure 1: Successive IGA approximations of the potential on the circular boundary, converging to the analytical solution

case $D o F$ is the number of the control coefficients of the NURBS approximation of the density $\mu(t)(D o F=n+\ell+1$; see eq. (13)). It can be clearly observed that the rate of convergence of the isogeometric method, $O\left(D o F^{-4}\right)$, is superior compared to that of the low-order panel method, $O\left(D o F^{-1}\right)$. An additional and interesting, from the practical point of view, way to compare the isogeometric approach versus low-order BEM is given in Fig. 3, depicting run time against error. Reported run times have been obtained employing MATLAB in a typical pc configuration with core- $2 \mathrm{CPU}$ at $2.4 \mathrm{GHz}$ with $2 \mathrm{~GB}$ RAM. It is worth noticing that, in the context of this example, IGA performs considerably better than low-order BEM. We can further increase the rate of convergence of the isogeometric method by using as initial NURBS representation of the circle a degree-elevated one ( $k$-refinement; see [Cottrell et al. 2007]) and then proceeding with $h$-refinement. This effect is illustrated in Fig. 4, where the $L^{\infty}$-norm of the error of the source density, as function of $D o F$, is depicted. In this figure the initial NURBS representation of the circle varies from quadratic to seventh degree while the rate of convergence is improved from $O\left(D o F^{-4}\right)$ to $O\left(D o F^{-10}\right)$ respectively. This dramatic improvement in the rate of convergence can be attributed to the fact that the, herein adopted, combined $k h$-refinement, provides higher-smoothness basis functions at a linear cost of $D o F$ increase.

In the second example we consider a free-form shape for $\partial \mathcal{D}$, as depicted in Fig.5. In this case, $\partial \mathcal{D}$ is represented

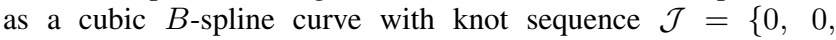
$0,0,1 / 8,1 / 4,3 / 8,1 / 2,5 / 8,3 / 4,7 / 8,1,1,1,1\}$ and control points: $[(-16,11.5),(-10,16.5),(4,1),(12,19),(32,17)$, $(1,-4),(29,-8),(2,-15),(-1,2),(-22,6.5),(16,11.5)]$.

The exterior flow considered is produced by two point sources of strength +1 and -1 (in order to have zero total flux through $\partial \mathcal{D}$ ) located inside the body at $(5,0)$ and $(10,5)$. Figure 6 is analogous to Figure 2 and reconfirms the superiority of the isogeometric versus the low-order panel method, giving nearly the same convergence rates. Since, in this case, we do not possess an explicit solution of the source density $\mu(t), t \in I$, analogous to eq. (35), Figure 7 depicts the $L^{\infty}$-norm of the relative error between successive approximations by using initial $B$-spline representation of varying degree from three to eight. In this case we have again a drastic improvement of the rate of convergence up to $O\left(D o F^{-9}\right)$ when 


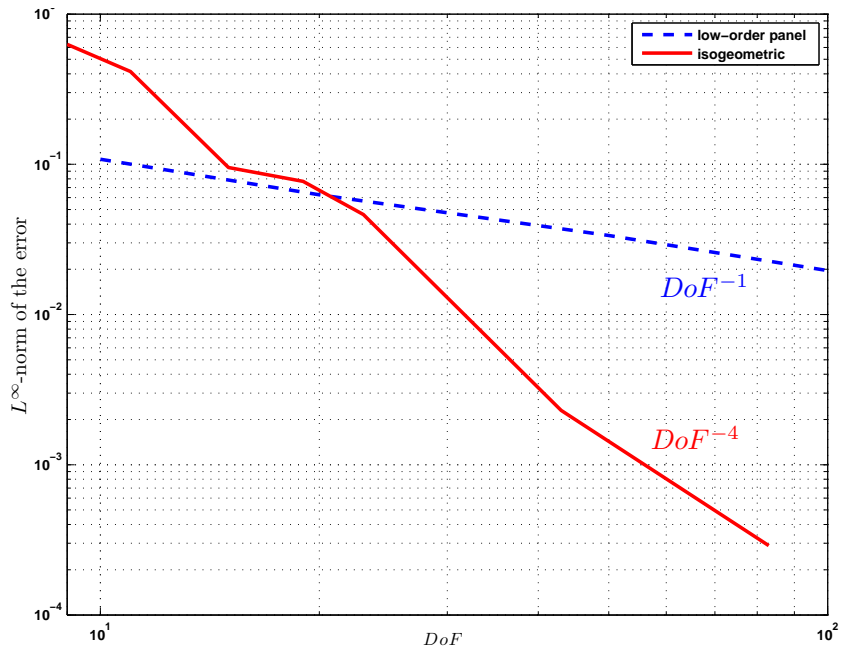

Figure 2: $L^{\infty}$-norm of the error of the potential on the circular boundary

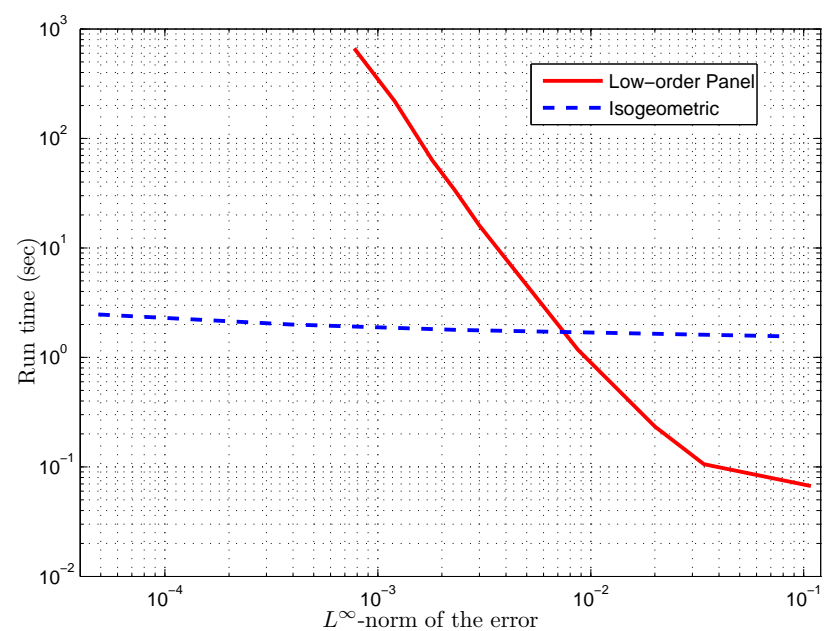

Figure 3: Required run-time versus $L^{\infty}$-norm of the error of the potential on the circular boundary

we use an $8^{t h}$ degree initial $B$-spline representation of $\partial \mathcal{D}$.

\section{Conclusions}

The isogeometric concept proposed by Hughes in the context of FEM is extended to BEM and employed to solve a classical exterior Neumann problem. Isogeometric BEM possesses all the advantages of the isogeometric concept (exact geometry representation, easy mesh refinement) and exhibits improved rates of convergence compared to classical BEM. The attained rate of convergence, when using knot insertion for refinement of the NURBS basis, is $O\left(D o F^{-4}\right)$, which is higher compared to low-order BEM but comparable to quadratic BEM. This rate can be further improved up to $O\left(D o F^{-9}\right)$ if knot insertion is suitably combined with degree elevation of the NURBS basis. On the ground of the encouraging results obtained so far, we intend to extend the method along two principal directions, involving non-smooth geometries, e.g. foils, as well as 3D immersed and/or floating bodies (the latter being a basic work package of the EXCITING project).

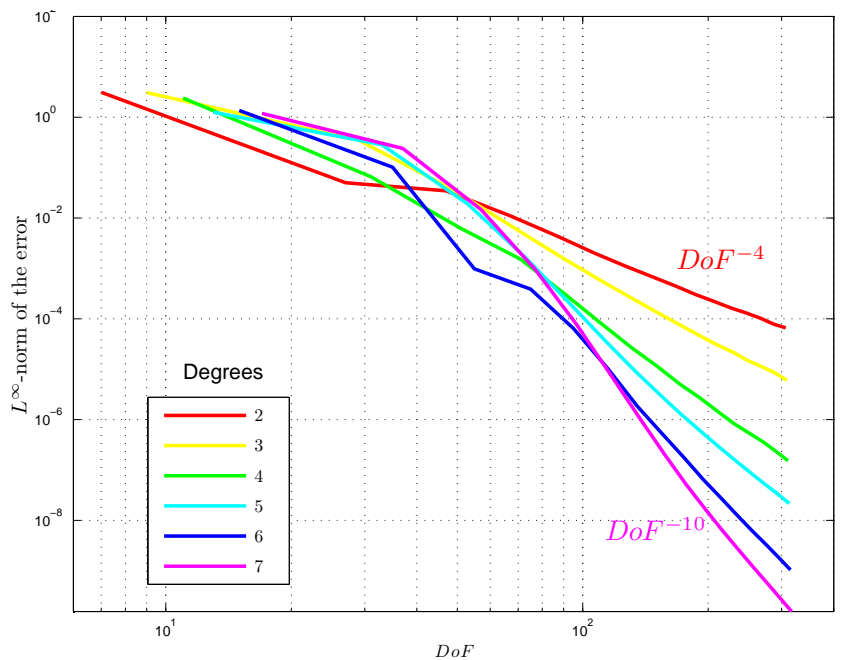

Figure 4: $L^{\infty}$-norm of the error of the source density for different degrees of the initial NURBS representation of the circle

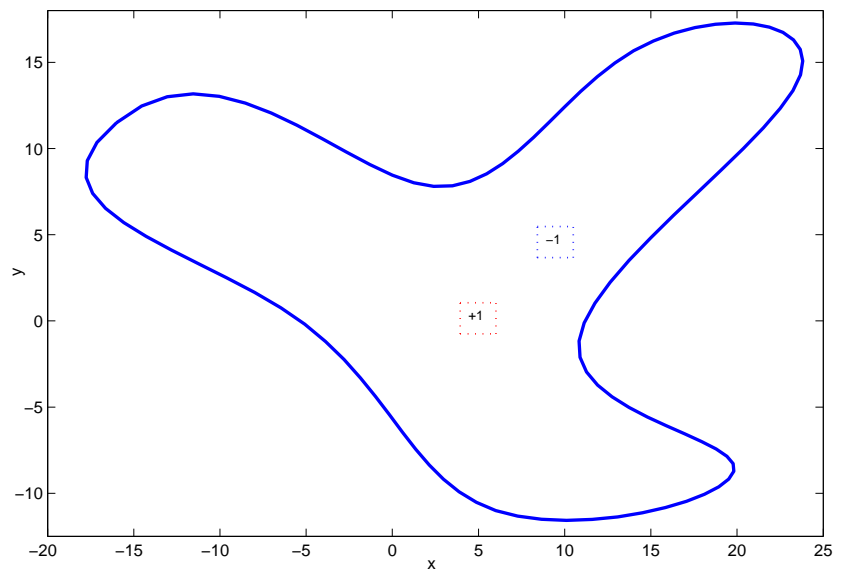

Figure 5: A free-form boundary along with the location and the strength of the Rankine singularities that generate the exterior flow field

\section{Acknowledgements}

The financial support by the FP7 EC-Project EXCITING (SCP82007-GA-218536) is gratefully acknowledged by all authors of the paper.

\section{References}

Brebiia, C., Telles, J., AND Wrobel, L. 1984. Boundary Element Techniques. Springer Verlag, Berlin.

BREBBiA, C. 2002. Recent innovations in BEM. Engineering Analysis with Boundary Elements 26.

Cottrell, J., Hughes, T., And Reali, A. 2007. Studies of refinement and continuity in isogeometric structural analysis. Computer Methods in Applied Mechanics and Engineering 196, 41-44, 4160-4183.

FARIN, G. 2001. Curves and surfaces for CAGD, a Practical Guide, 5th Edition. Morgan Kaufmann Publishers. 


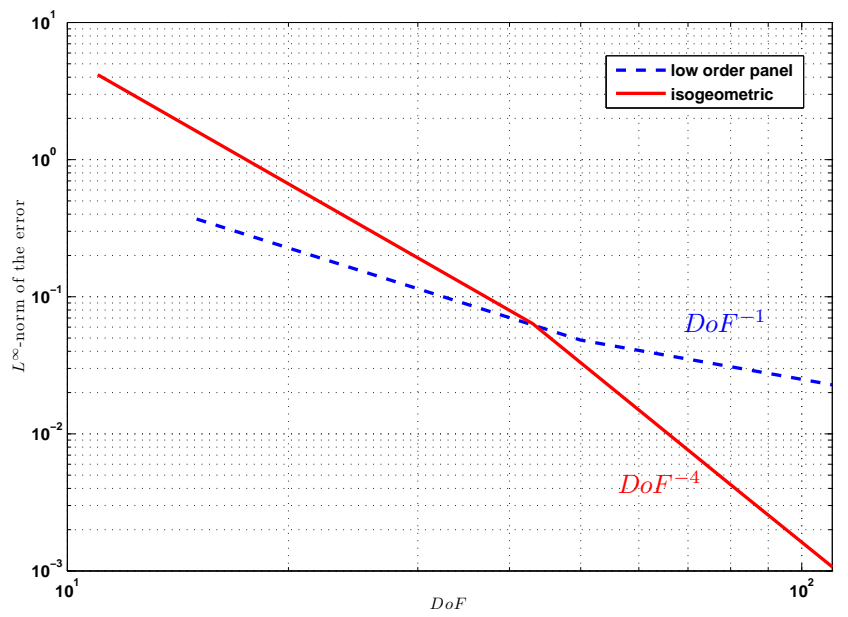

Figure 6: $L^{\infty}$-norm of the error of the potential on the free-form boundary

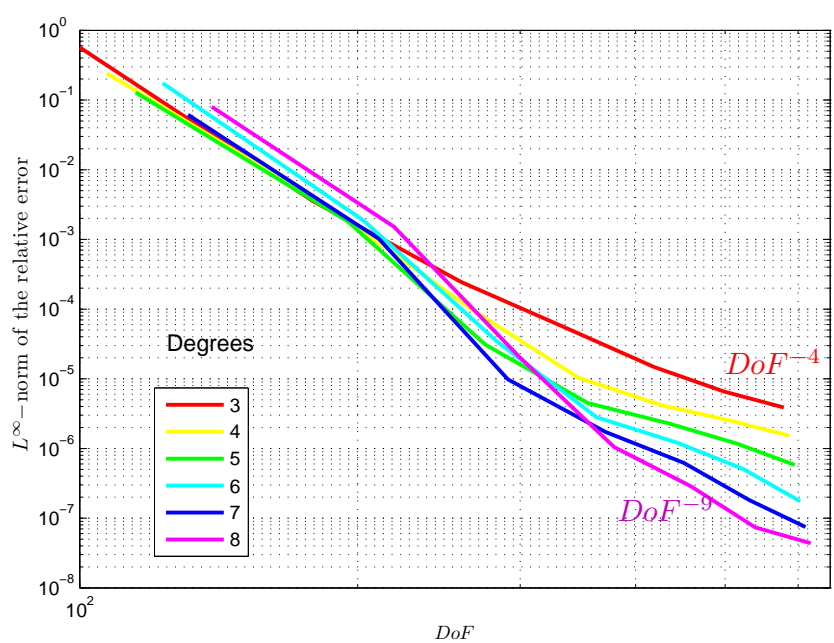

Figure 7: $L^{\infty}$-norm of the relative error of the source density for different degrees of the initial NURBS representation of the freeform boundary

HESS, J. 1975. Improved solution for potential flow about axisymmetric bodies by use of a higher order surface source method. Comp. Meth. Appl. Mech. Eng. 5, 297-308.

Hughes, T., Cottrell, J., AND Bazilevs, Y. 2005. Isogeometric analysis: CAD, finite elements, NURBS, exact geometry and mesh refinement. Comput. Methods Appl. Mech. Engrg. 194, 4135-4195.

HUGHES, T. (to appear). Isogeometric analysis: Progress and challenges. In Proceedings of the International Conference on Mathematical Methods for Curves and Surfaces (MMCS'08).

JAWSON, M., AND SYMM, G. 1977. Integral equation methods in potential theory and elastostatics. Academic Press.

Kress, R. 1989. Linear Integral Equations. Springer Verlag, Berlin.

Krishnaswamy, P., Andersen, P., and Kinnas, S. 2001. Re-entrant jet modelling for partially cavitating two-dimensional hydrofoils. In Proceedings of Cav2001.
Milne-Thomson, L. 1956. Theoretical Hydrodynamics. Macmillan Company, New York.

Paris, F., AND CANAS, J. 1997. Boundary Element Methods. Oxford University Press.

Piegl, L., AND TILler, W. 1997. The NURBS Book, 2nd Edition. Springer Verlag.

SChMidt, G., And Strese, H. 2001. The convergence of a direct BEM for the plane mixed boundary value problem of the Laplacian. Numerische Mathematik 54, 145-165.

SladeK, V., SladeK, J., And Tanaka, M. 2001. Numerical integration of logarithmic and nearly logarithmic singularity in BEMs. Applied Mathematical Modeling 25, 901-922.

VAZ, G., DE CAmpos, J. F., AND ECA, L. 2003. A numerical study on low and higher-order potential based BEM for $2 \mathrm{~d}$ inviscid flows. Computational Mechanics 32, 327-335. 